\section{Pólos de produção de leishmaniose tegumentar americana no norte do Estado do Paraná, Brasil}

\author{
Poles of American tegumentary leishmaniasis \\ production in northern Paraná State, Brazil
}

\author{
1 Programa de Pós-gradu- \\ ação em Medicina Tropical, \\ Universidade do Estado \\ do Amazonas/Fundação \\ de Medicina Tropical do \\ Amazonas, Manaus, Brasil. \\ 2 Centro de Ciências \\ Biológicas e da Saúde, \\ Universidade Estadual do \\ Oeste do Paraná, Cascavel, \\ Brasil. \\ 3 Departamento de Análises \\ Clínicas, Universidade \\ Estadual de Maringá, \\ Maringá, Brasil. \\ ${ }^{4}$ Departamento de Geografia, \\ Universidade Estadual de \\ Maringá, Maringá, Brasil. \\ Correspondência \\ W. M. Monteiro \\ Programa de Pós-gradu- \\ ação em Medicina Tropical, \\ Universidade do Estado \\ do Amazonas/Fundação \\ de Medicina Tropical do \\ Amazonas. \\ Av. Pedro Teixeira 25, \\ Manaus, $A M$ \\ 69040-000, Brasil. \\ wueltonmm@ibest.com.br
}

\begin{abstract}
American tegumentary leishmaniasis is endemic in the State of Paraná, with 99.3\% of the cases reported in the South of Brazil. Spatial distribution of the disease in northern Paraná was verified, identifying the most relevant geographic areas in epidemiological terms. The study used data recorded on epidemiological forms from the Teaching and Research Clinical Test Laboratory of the State University in Maringá, from 1987 to 2004. The study only included individuals that were infected in the municipalities (counties) in northern Paraná. Identification of the epidemiological units (poles and circuits) was based on spatial density of cases, according to the model proposed by the National Health Foundation, considering the most likely infection sites. Considering 1,933 reported cases, 1,611 were infected in northern Paraná. American tegumentary leishmaniasis distribution in Paraná State suggests two circuits for production of the disease: Paraná-Paranapanema, highlighting the Cinzas-Laranjinha, Tibagi, Ivaí-Pirapó, Piquiri, and Baixo Iguaçu poles, and Ribeira, highlighting the Alto Ribeira pole.
\end{abstract}

Leishmaniasis; Endemic Diseases; Communicable Diseases

\author{
Wuelton Marcelo Monteiro ${ }^{1}$ \\ Herintha Coeto Neitzke 2 \\ Thaís Gomes Verzignassi Silveira ${ }^{3}$ \\ Maria Valdrinez Campana Lonardoni 3 \\ Ueslei Teodoro ${ }^{3}$ \\ Maria Eugênia Moreira Costa Ferreira 4
}

\section{Introdução}

A leishmaniose tegumentar americana ocorre nas Américas, desde o Sul dos Estados Unidos até o Norte da Argentina. A incidência desta doença vem aumentando na América Latina, especialmente no Brasil, que registrou 605.062 casos, de 1980 a 2005 1. Neste período, na Região Sul, notificaram-se 13.384 casos, dos quais $13.206(98,7 \%)$ no Estado do Paraná ${ }^{1}$, particularmente no norte e oeste ${ }^{2}$.

O padrão de transmissão da leishmaniose tegumentar americana no Estado do Paraná tem vínculo com o ciclo silvestre do parasito em focos naturais que persistem em áreas florestais preservadas como rugosidades em zonas de produção agropecuária tradicional. As áreas que se destacam pela densidade intensa de casos localizam-se, sobretudo, nas bacias dos rios Ivaí e Pirapó, onde ainda há grandes manchas de florestas residuais medianamente ou muito alteradas e florestas secundárias 2,3. As ações antrópicas no ambiente, a urbanização crescente e as pressões sócio-econômicas têm expandido as áreas endêmicas e o aparecimento de focos da leishmaniose tegumentar americana em zonas urbanas ${ }^{4} \mathrm{Na}$ zona urbana, a doença tem ocorrido em áreas com preservação de pequenos trechos de cobertura florestal, a exemplo dos municípios de Maringá 2,5 e Cianorte 2. No Paraná, a leishmaniose tegumentar americana persiste, mesmo com a substituição da vegetação original de florestas 
pelas culturas de café, soja, milho, algodão e por pastagens, afetando indivíduos de todos os grupos etários e de ambos os sexos 2,3.

As infecções humanas no Paraná são causadas principalmente pela espécie Leishmania braziliensis ${ }^{6}$. As espécies de flebotomíneos predominantes são Nyssomyia whitmani e Nyssomyia neivai 5,7,8,9,10. Esses flebotomíneos têm sido coletados em grande número em zonas rurais onde as habitações têm condições precárias de higiene, há acúmulo de matéria orgânica no peridomicílio, a umidade do solo é elevada e os abrigos de animais domésticos são próximos do domicílio, observando-se ainda a presença de matas remanescentes de tamanhos variados na proximidade das residências 5,7,8,9,10,11,12 .

O modelo de vigilância e monitoramento da leishmaniose tegumentar americana em unidades territoriais, proposto pela Fundação Nacional de Saúde 13, identifica dois circuitos de produção da doença no Estado do Paraná, denominados Grande Região de Londrina e Vale do Paranapanema. O primeiro abrange especialmente os municípios das mesorregiões norte central e do Norte Pioneiro Paranaense e o segundo das mesorregiões, centro ocidental e noroeste. Contudo, há diversas falhas na notificação (fichas de notificação inadequadas; preenchimento incompleto; erros no preenchimento etc.) 2,14, além da concentração de notificações da doença nos municípios-sede das regionais de saúde da Secretaria de Saúde do Estado do Paraná 2 , o que dificulta o conhecimento da distribuição geográfica da leishmaniose tegumentar americana e das implicações epidemiológicas decorrentes das mudanças ecológicas induzidas pelas ações antrópicas no ambiente natural. Este conhecimento é essencial para a compreensão do padrão de transmissão da leishmaniose tegumentar americana e para subsidiar o estabelecimento de medidas adequadas para o controle desta doença. Assim, o objetivo deste trabalho é caracterizar as unidades espaciais (municípios) de importância epidemiológica na produção autóctone de leishmaniose tegumentar americana nas mesorregiões supracitadas, no Estado do Paraná, Sul do Brasil.

\section{Materiais e métodos}

\section{Descrição das mesorregiões}

As mesorregiões norte central, centro ocidental e noroeste do Estado do Paraná situam-se entre os meridianos de 5130' e 5400' longitude oeste e os paralelos de $22^{\circ} 30^{\prime}$ e $24^{\circ} 35^{\prime}$ latitude sul, abrangendo 162 municípios, numa área de $61.250,8 \mathrm{~km}^{2}$ e uma população de 2.816 .800 habi- tantes, com $2.364 .414(83,9 \%)$ na zona urbana e $452.386(16,1 \%)$ na zona rural (Instituto Brasileiro de Geografia e Estatística. Censo Demográfico de 2000. http://www.ibge.gov.br, acessado em 05/Fev/2005). As mesorregiões supracitadas, em conjunto com o oeste de São Paulo e sul do Mato Grosso do Sul, foram ocupadas durante a primeira metade do século XX, constituindo zonas agrícolas pioneiras do Brasil 15 .

A vegetação nativa, na sua maior parte destruída, era constituída por floresta estacional semidecidual montana e submontana, com queda parcial das folhas de certas espécies arbóreas no inverno, quando ocorre seca pouco pronunciada. No Paraná, as raras manchas dessa floresta tropical ou subtropical de transição encontramse, ainda, precisamente nas margens dos rios Ivaí, Paranapanema, Paraná e de seus afluentes. O clima é tropical de transição para subtropical, apresentando temperaturas médias anuais entre $20^{\circ} \mathrm{C} \mathrm{e} 22^{\circ} \mathrm{C}$, com médias do mês mais quente superior a $22^{\circ} \mathrm{C}$, e invernos brandos com períodos de seca hibernal pouco pronunciada, dos meses de julho a setembro 16. A precipitação anual avaliada por um período de cinco anos indica média de $1.600 \mathrm{~mm} /$ ano, com menores valores nas calhas dos rios Paranapanema e Paraná, sendo novembro o mês mais chuvoso e agosto o mais seco 16 .

As mesorregiões abrangidas no presente estudo pertencem ao Terceiro Planalto Paranaense, constituído por derrames de lavas básicas intercaladas em estratos de arenito, cuja inclinação decai suavemente para oeste, em direção ao rio Paraná 16 . A paisagem, no seu conjunto, é de planalto levemente ondulado, com amplos interflúvios e vales abertos, embora os rios possam ocorrer em leitos por vezes encaixados, como é o caso dos rios Paraná e Ivaí, em alguns trechos. Os terrenos onde aflora a cobertura suprabasáltica (Arenito Caiuá) e outras formações cenozóicas, que sucederam o derrame de lava do Mesozóico, apresentam relevo mais aplainado, em mesetas, tal como ocorre no interflúvio Pirapó-Bandeirantes do Norte e no divisor Ivaí-Piquiri 16.

A maioria dos municípios dessas mesorregiões está classificada no nível médio inferior de desenvolvimento humano, tendo o setor primário como o mais importante, quanto à participação setorial no valor adicionado fiscal (a agropecuária extensiva contribui com a maior parcela do valor bruto da produção silvoagropecuária) 17 . A maioria dos municípios vem apresentando taxas negativas de crescimento da população total, desde 1970 até 2000, e o crescimento geométrico da população rural, entre 1991 e 2000, foi negativo nas três mesorregiões e positivo para a população urbana, no mesmo período. 
As mesorregiões do estudo são aquelas com o maior grau de desigualdade de renda no Estado do Paraná 11 .

\section{Procedimentos}

O estudo foi realizado com dados registrados em fichas epidemiológicas do Laboratório de Ensino e Pesquisa em Análises Clínicas da Universidade Estadual de Maringá (LEPAC/UEM), centro de referência para leishmaniose tegumentar americana no Sul do Brasil, de 1987 a 2004. Os dados coletados para a realização deste trabalho foram: município onde provavelmente ocorreu a infecção, localidade mais provável de infecção, sexo e moradia (rural/urbana). Os pacientes foram considerados positivos quando tiveram pelo menos um dos seguintes testes diagnósticos positivo: pesquisa direta de Leishmania, imunofluorescência indireta (título $\geq 40$ ) ou reação intradérmica de Montenegro (diâmetro $\geq 5 \mathrm{~mm}$ ).

Para a caracterização preliminar das unidades territoriais de relevância epidemiológica (pólos e circuitos) utilizou-se a metodologia proposta pela Fundação Nacional de Saúde 13. Os casos foram mapeados de acordo com as prováveis localidades de infecção e foram calculadas as densidades de casos por quilômetro quadrado e o coeficiente de detecção da doença (casos/100 mil habitantes), tomando-se o município como unidade de análise. Segundo a Fundação Nacional de Saúde 13 , o circuito de produção de leishmaniose tegumentar americana compreende uma região extensa, complexa e contínua, caracterizada pela elevada produção de casos, constituído pelos pólos e pelas áreas com casos isolados; os pólos, por sua vez, são unidades espaciais, com determinado número de municípios, que se destacam por sua densidade intensa de casos produzidos, em contraste com as áreas vizinhas.

Para se avaliar as condições facilitadoras da produção da leishmaniose tegumentar americana, verificou-se a distribuição geográfica da doença em corredores da hidrografia de áreas originalmente cobertas pela floresta estacional e da hidrografia de áreas cobertas pela floresta Atlântica.

\section{Resultados}

Foram identificados dois pólos de produção de leishmaniose tegumentar americana, separados pelo interflúvio das bacias dos rios Pirapó e Ivaí (Figura 1).

No período de 1987 a 2004 foram diagnosticados 1.938 casos de leishmaniose tegumentar americana, provenientes de municípios inseridos na região do estudo, dos quais $1.761(91,1 \%)$ informaram o município onde ocorreu a infecção. Destes, 1.611 (91,5\%) indivíduos se infectaram nas mesorregiões referidas e 150 (8,5\%) em outros estados ou no Paraguai. Dos 1.611 casos com infecção em território paranaense, os homens representaram $1.180(73,2 \%)$ casos e as mulheres 431 (26,8\%); 1.493 (92,7\%) indivíduos se infectaram na zona rural e 118 (7,3\%) na zona urbana.

Os municípios paranaenses com maior número de casos registrados foram São Jorge do Ivaí com 181 (11,2\%), Doutor Camargo com 153 (9,5\%), Maringá com 143 (8,9\%), Terra Boa com 127 (7,9\%), Jussara com 101 (6,3\%), Cianorte com 91 (5,6\%), Colorado com 87 (5,4\%) e Lobato com $82(5,1 \%)$ casos, que juntos perfizeram $59,9 \%$ do total de indivíduos que se infectaram nas mesorregiões em estudo (Tabela 1). No entanto, os municípios com as maiores densidades de casos foram Doutor Camargo (1,304), São Jorge do Ivaí $(0,473)$, Jussara $(0,463)$, Ivatuba $(0,406)$, Terra Boa $(0,400)$, Lobato $(0,350)$, Maringá $(0,294)$ e Uniflor $(0,251)$ (Tabela 1). Verificou-se que os municípios com maior número de casos urbanos autóctones foram Maringá (36), Cianorte (18) e Terra Boa (8).

Identificaram-se as localidades mais prováveis onde ocorreram as infecções de 718 casos. Em 54 municípios foram identificadas 308 localidades como focos de leishmaniose tegumentar americana, e destas, em 75 foram notificados mais de um caso, destacando-se a Fazenda Palmital, Município de Terra Boa (74 casos); o Distrito de Copacabana do Norte, Município de São Jorge do Ivaí (38 casos); a Fazenda Jussara, Município de Jussara (35 casos); Distrito de Iguatemi, Município de Maringá (25 casos); a Fazenda da Barra, Município de Lobato (18 casos); a Olaria Andirá, Município de Jussara (18 casos); a Fazenda Remanso Município de Lobato (17 casos); o Porto Andirá, Município de Jussara (17 casos); o Recanto Marista, Município de Doutor Camargo (12 casos); a Fazenda da Reserva, Município de Doutor Camargo (11 casos); e o Distrito de Alto Alegre, Município de Colorado (10 casos). Esses focos, juntos, perfazem $38,3 \%$ dos casos, nas localidades com maior probabilidade de ocorrência de infecção. Os municípios com o maior número de focos foram São Jorge do Ivaí (29), Maringá (28), Doutor Camargo (22), Mandaguaçu (20), Nova Esperança (19), Marialva (18), Colorado (17) e Terra Boa (16).

Os coeficientes de detecção em cinqüenta municípios foram baixos ( 0 a 3), em 22 foram médios ( 3 a 11) e em 20 foram altos (11 a 70). Os municípios de Doutor Camargo $(118,8)$, São Jorge do Ivaí $(116,5)$ e Lobato $(100,0)$ apresentaram coeficientes muito altos (>70) (Tabela 1). 


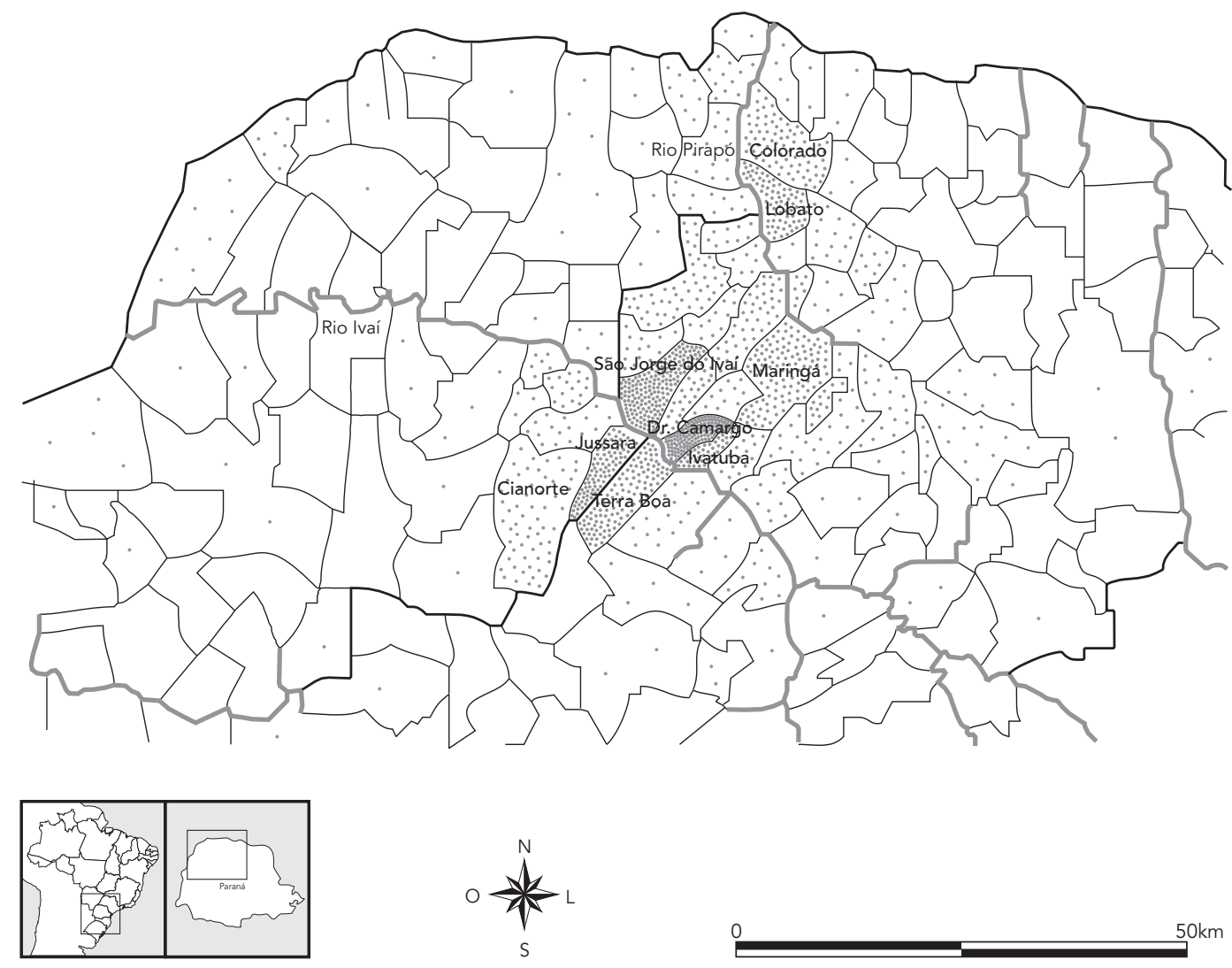

Nota: 1 ponto -1 caso

\section{Discussão}

A leishmaniose tegumentar americana apresentou um aumento expressivo no número de casos a partir das duas últimas décadas do século passado, assim como uma importante difusão espacial ${ }^{13}$. A doença vem sendo registrada em áreas de ocupação antiga, inclusive em espaços urbanos, contrariando a expectativa de que, com o aumento da pressão antrópica e a conseqüente eliminação dos focos naturais, esta endemia deveria ter sua importância reduzida 18 . No Estado do Paraná a distribuição geográfica da doença é ampla e irregular, com concentração de casos em municípios do norte e oeste desse estado, em áreas que sofreram intensa antropia 2,3.

A ocorrência de casos autóctones de leishmaniose tegumentar americana em todos os anos do período de 1987 a 2003, em diversos municípios das mesorregiões norte central, centro ocidental e noroeste, revela o caráter endêmico e o quão ampla é a dispersão dessa doença nos municípios das mesorregiões referidas 3,4,6, onde identificam-se dois pólos de produção da doença. Um ao norte (pólo Paranapanema-Pirapó), abrangendo os municípios banhados pelos rios Paranapanema (Paranapoema, Jardim Olinda, Itaguajé, Santa Inês e Santo Inácio) e Pirapó (Colorado, Lobato e Uniflor). Outro mais ao sul (pólo Ivaí), abrangendo municípios pertencentes à bacia do rio Ivaí (Doutor Camargo, São Jorge do Ivaí, Jussara, Ivatuba, Terra Boa, Ourizona, Cianorte, Fênix, Japurá, São Tomé). Os dois pólos pertencem ao circuito Paranapanema de produção de leishmaniose tegumentar americana 13. A densidade de casos é notável nesses dois pólos, mas o pólo Ivaí é o mais importante por concentrar grande nú- 
Número, densidade espacial e coeficiente de detecção dos casos de leishmaniose tegumentar americana, conforme municípios onde ocorreram as infecções, diagnosticados no Laboratório de Ensino e Pesquisa em Análises Clínicas da Universidade Estadual de Maringá (LEPAC/UEM), de 1987 a 2004.

\begin{tabular}{|c|c|c|c|c|c|c|c|}
\hline Município & Casos & $\begin{array}{c}\text { Densidade } \\
\text { de casos } \\
\text { por km² }\end{array}$ & $\begin{array}{l}\text { Coeficiente } \\
\text { de detecção } \\
\text { (casos/100 mil } \\
\text { habitantes) }\end{array}$ & Município & Casos & $\begin{array}{c}\text { Densidade } \\
\text { de casos } \\
\text { por km² }\end{array}$ & $\begin{array}{l}\text { Coeficiente } \\
\text { de detecção } \\
\text { (casos/100 mil } \\
\text { habitantes) }\end{array}$ \\
\hline Alto Paraná & 3 & 0,007 & 1,3 & Mandaguari & 23 & 0,068 & 4,7 \\
\hline Ângulo & 7 & 0,067 & 15,8 & Marialva & 32 & 0,067 & 9,9 \\
\hline Apucarana & 12 & 0,022 & 0,8 & Marilena & 1 & 0,004 & 0,8 \\
\hline Arapongas & 6 & 0,016 & 0,5 & Mariluz & 1 & 0,003 & 0,5 \\
\hline Assai & 2 & 0,004 & 0,6 & Maringá & 143 & 0,294 & 9,9 \\
\hline Astorga & 16 & 0,036 & 3,9 & Marumbi & 1 & 0,005 & 1,1 \\
\hline Atalaia & 6 & 0,045 & 9,5 & Munhoz de Melo & 9 & 0,065 & 15,6 \\
\hline Bandeirantes & 1 & 0,002 & 0,2 & Nossa Senhora das Graças & 5 & 0,030 & 9,0 \\
\hline Barbosa Ferraz & 1 & 0,002 & 0,7 & Nova Esperança & 53 & 0,149 & 15,8 \\
\hline Bom Sucesso & 2 & 0,006 & 3,3 & Ourizona & 27 & 0,154 & 52,3 \\
\hline Borrazópolis & 2 & 0,006 & 1,4 & Paiçandu & 22 & 0,157 & 16,4 \\
\hline Califórnia & 1 & 0,007 & 0,7 & Paraíso do Norte & 1 & 0,005 & 0,6 \\
\hline Cambira & 4 & 0,024 & 2,1 & Paranacity & 13 & 0,032 & 9,5 \\
\hline Campina da Lagoa & 1 & 0,001 & 0,3 & Paranapoema & 11 & 0,094 & 11,5 \\
\hline Campo Mourão & 2 & 0,003 & 0,2 & Paranavaí & 6 & 0,005 & 0,4 \\
\hline Cianorte & 91 & 0,113 & 10,2 & Peabiru & 4 & 0,008 & 0,8 \\
\hline Cidade Gaúcha & 2 & 0,005 & 0,6 & Pérola & 1 & 0,004 & 0,5 \\
\hline Colorado & 87 & 0,211 & 26,6 & Pitanga & 1 & 0,001 & 0,1 \\
\hline Corbélia & 1 & 0,002 & 0,3 & Porto Rico & 7 & 0,030 & 1,9 \\
\hline Corumbataí do Sul & 2 & 0,012 & 1,9 & Presidente Castelo Branco & 15 & 0,094 & 27,8 \\
\hline Cruzeiro do Sul & 10 & 0,046 & 11,3 & Querência do Norte & 7 & 0,008 & 3,6 \\
\hline Cruzmaltina & 1 & 0,003 & 1,6 & Quinta do Sol & 4 & 0,012 & 3,9 \\
\hline Diamante do Norte & 1 & 0,005 & 0,8 & Roncador & 1 & 0,001 & 0,4 \\
\hline Doutor Camargo & 153 & 1,304 & 118,8 & Rondon & 3 & 0,006 & 2,5 \\
\hline Engenheiro Beltrão & 18 & 0,038 & 4,2 & Santa Fé & 17 & 0,060 & 12,6 \\
\hline Fênix & 9 & 0,040 & 9,1 & Santa Inês & 2 & 0,015 & 2,6 \\
\hline Floraí & 20 & 0,096 & 24,5 & Santo Antônio do Caiuá & 2 & 0,009 & 2,7 \\
\hline Floresta & 18 & 0,114 & 16,2 & Santo Inácio & 5 & 0,019 & 5,2 \\
\hline Flórida & 4 & 0,049 & 9,9 & São Carlos do Ivaí & 4 & 0,016 & 1,0 \\
\hline Formosa do Oeste & 2 & 0,007 & 1,0 & São João do Caiuá & 1 & 0,003 & 0,9 \\
\hline Goioerê & 1 & 0,002 & 0,2 & São João do Ivaí & 1 & 0,003 & 0,4 \\
\hline Icaraíma & 1 & 0,001 & 0,5 & São Jerônimo da Serra & 1 & 0,001 & 0,4 \\
\hline Iguaraçu & 5 & 0,032 & 7,7 & São Jorge do Ivaí & 181 & 0,473 & 116,5 \\
\hline Inajá & 8 & 0,041 & 8,0 & São Jorge do Patrocínio & 1 & 0,003 & 0,7 \\
\hline Indianópolis & 3 & 0,023 & 3,2 & São Manoel do Paraná & 6 & 0,064 & 2,7 \\
\hline Itaguajé & 4 & 0,019 & 3,4 & São Pedro do Ivaí & 1 & 0,004 & 0,6 \\
\hline Itambé & 15 & 0,062 & 13,7 & São Pedro do Paraná & 1 & 0,005 & 1,8 \\
\hline Ivaté & 1 & 0,002 & 0,8 & São Tomé & 17 & 0,067 & 16,1 \\
\hline Ivatuba & 38 & 0,406 & 69,3 & Sarandi & 15 & 0,203 & 8,8 \\
\hline Jandaia do Sul & 3 & 0,016 & 1,8 & Tapejara & 1 & 0,002 & 1,3 \\
\hline Japurá & 16 & 0,105 & 15,2 & Terra Boa & 127 & 0,400 & 43,6 \\
\hline Jardim Olinda & 2 & 0,015 & 7,3 & Terra Rica & 1 & 0,001 & 0,4 \\
\hline Jesuítas & 1 & 0,004 & 0,5 & Toledo & 1 & 0,001 & 0,1 \\
\hline Jussara & 101 & 0,463 & 45,2 & Tuneiras do Oeste & 1 & 0,001 & 0,6 \\
\hline Loanda & 1 & 0,001 & 0,3 & Umuarama & 2 & 0,001 & 0,1 \\
\hline Lobato & 82 & 0,350 & 100,0 & Uniflor & 14 & 0,251 & 37,5 \\
\hline Londrina & 2 & 0,001 & 0,1 & Vila Alta & 4 & 0,004 & 5,9 \\
\hline Mandaguaçu & 45 & 0,154 & 25,2 & Total & 1.611 & - & 10,9 \\
\hline
\end{tabular}


mero de casos, em diversos municípios e focos endêmicos.

Como a produção da doença é mais intensa nos municípios da bacia hidrográfica do rio Ivaí, que não tem relação geográfica direta com a bacia do rio Paranapanema, considera-se que a denominação Circuito Vale do Paranapanema 13 não condiz com a real área de abrangência do mesmo, pois os municípios pertencentes ao pólo Paranapanema-Pirapó apresentam menor incidência de leishmaniose tegumentar americana do que aqueles pertencentes ao pólo Ivaí. Da mesma forma, a incidência dessa doença nos municípios banhados pelo rio Pirapó é superior à incidência nos municípios banhados pelo rio Panapanema. Desse modo, a denominação mais apropriada seria Ivaí-Pirapó para o referido circuito. Chama a atenção o fato de que não há limites nítidos entre os circuitos Vale do Paranapanema e Grande Região de Londrina, onde em diversos municípios 13 foram registrados numerosos casos da doença 2,19,20. Além disso, a contigüidade entre estas unidades epidemiológicas sugere a formação de um único circuito de produção (Figuras 1 e 2), conforme o conceito de circuito da Fundação Nacional de Saúde 13. Na Figura 2, verifica-se que há dificuldades para distinguir os limites dos circuitos referidos, pois no norte, oeste e leste do Paraná há grandes concentrações de casos de leishmaniose tegumentar americana. Outrossim, considerando-se o conceito de circuito de produção da doença 13,

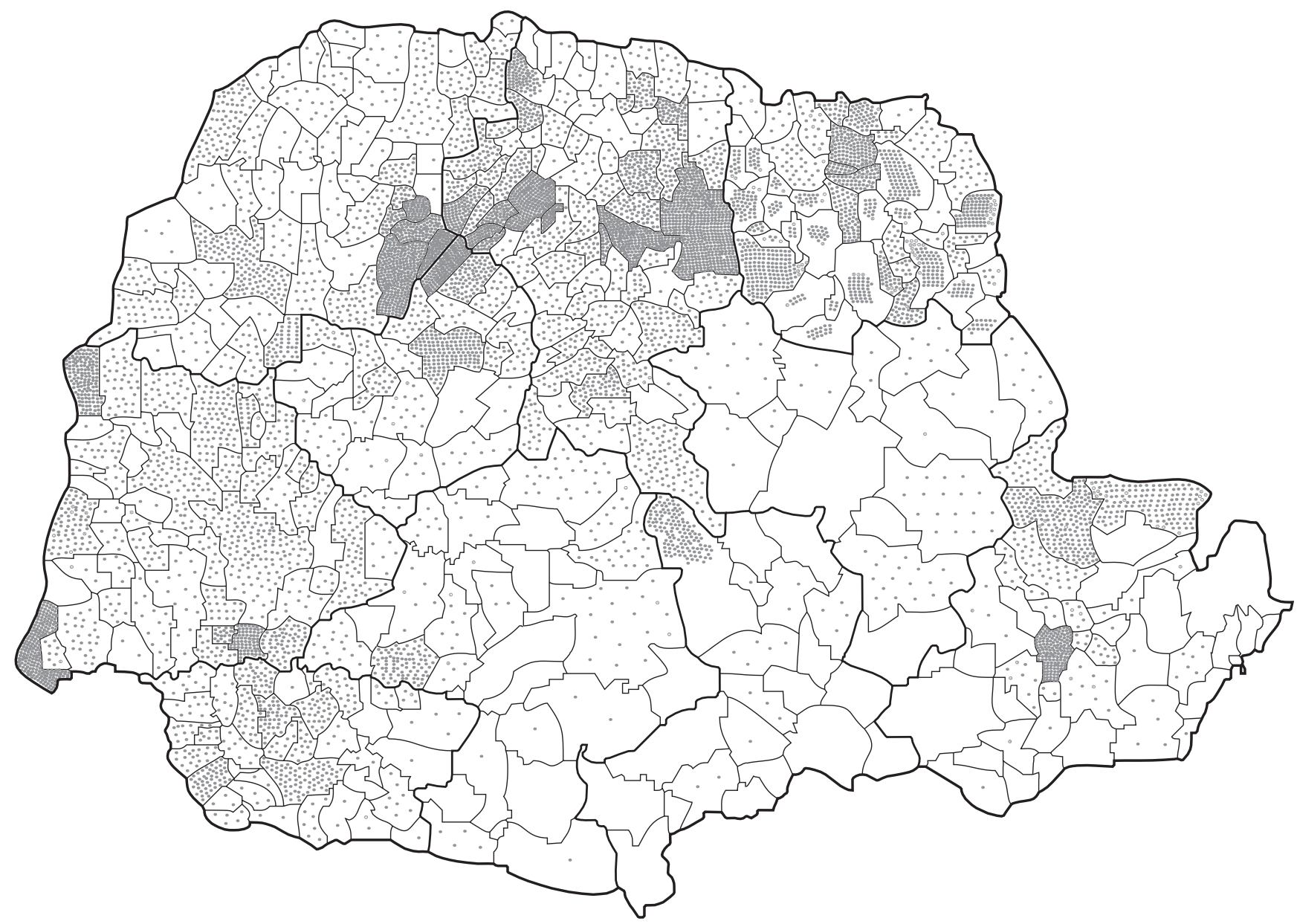

Nota: 1 ponto -1 caso 
e mediante os fatos relatados, propõe-se que todo o Estado do Paraná seja considerado endêmico, com dois circuitos: Paraná-Paranapanema e Ribeira. O primeiro, com pólos em corredores da hidrografia de áreas originalmente cobertas pela floresta estacional, da qual restam apenas remanescentes, destacando-se os pólos CinzasLaranjinha, Tibagi, Ivaí-Pirapó, Piquiri e Baixo Iguaçu; e o segundo, em corredores da hidrografia de áreas cobertas pela floresta atlântica, onde se destaca o pólo Alto Ribeira.

As imagens obtidas por sensoriamento remoto orbital mostram que no pólo Ivaí a leishmaniose tegumentar americana tem relação muito íntima com áreas de mata nativa modificada e pequena florestas ciliares nas margens do rio Ivaí e dos seus afluentes ${ }^{2}$, onde tem sido constatada elevada freqüência de flebotomíneos, destacando-se N. whitmani, N. neivai e Migonemyia migonei 7,8,11,12. Nos municípios de Jussara e Terra Boa, que apresentaram casos em todos os anos do estudo, existem registros de focos da doença desde o final da década de 1970, quando a notificação ainda não era obrigatória 21 . No último triênio, observou-se um aumento no número de casos nos municípios de Japurá, São Tomé e São Manoel do Paraná e, particularmente em Cianorte, indicando uma expansão geográfica da doença. Nesse contexto, a leishmaniose tegumentar americana persiste, mesmo com a substituição da vegetação original de florestas pelas culturas de café, soja, milho, algodão e por pastagens 2, afetando cães 22,23 e humanos de todos os grupos etários e de ambos os sexos 2,3,6.

No pólo Pirapó, onde se destacam os focos da Fazenda da Barra e da Fazenda Remanso, no Município de Lobato, os casos estão majoritariamente relacionados às reservas de mata nativa preservadas nas margens do rio Pirapó, onde também tem sido constatada uma grande freqüência de flebotomíneos, sobretudo das espécies $N$. whitmani e $N$. neivai 9,10. Os casos que incidiram sobre trabalhadores temporários nas fazendas supracitadas representam grande parte dos casos deste pólo ${ }^{3}$.

A maioria dos indivíduos se infectou na zona rural, com predomínio do sexo masculino, o que foi observado anteriormente no Estado do Paraná 3,6. Apesar desses resultados, não se pode afirmar que o risco está ligado ao trabalho agrícola, já que em trabalho anterior a proporção de pessoas ocupadas com atividades domésticas foi semelhante àquela das pessoas envolvidas com o trabalho rural ${ }^{3}$. O considerável contingente de mulheres e crianças com leishmaniose tegumentar americana, demonstrado em alguns estudos realizados no Paraná, corrobora tal afirmação 3,6. Vale salientar que uma grande proporção dos ca- sos, principalmente do sexo masculino, que adquiriram a infecção no ambiente rural, residiam na zona urbana. Para estes casos, a mobilidade pendular, referente ao trabalho rural temporário, representa importante fator de risco para a aquisição da infecção ${ }^{3}$. Os 150 casos com infecção em outros estados ou do Paraguai demonstram, novamente, que a mobilidade populacional intervém na epidemiologia da leishmaniose tegumentar americana, no Paraná.

O coeficiente médio anual de detecção de casos de leishmaniose tegumentar americana por 100 mil habitantes foi de 10,9, em 95 municípios do Estado do Paraná. Em estudo anterior, realizado em municípios atendidos pela Regional de Saúde de Arapongas 20, na mesorregião norte central do estado, de 1993 a 1998, o coeficiente de detecção foi menor $(4,81)$ do que no presente trabalho. No Estado do Paraná, o coeficiente de detecção foi 4,88 no ano de 1999 24. Os valores encontrados são inferiores à média do Brasil (18,63 em 1999) 24 . Estes resultados refletem a intensa variação temporal na incidência da leishmaniose tegumentar americana nas mesmas localidades e municípios de uma mesma área endêmica, dependendo de diversos fatores, que estão por ser elucidados. Na área do presente estudo verificaram-se coeficientes de detecção tão altos como 118,8, 116,5 e 100,0, respectivamente nos municípios de Doutor Camargo, São Jorge do Ivaí e Lobato, comparáveis aos observados em estados com incidência muito alta da doença 24 . Em 2005, os coeficientes de incidência, por 100 mil habitantes, de Cianorte e Jussara foram 35,4 e 355,9, e no Brasil e Paraná foram 13,2 e 3,9, respectivamente 25 .

É possível que o coeficiente de detecção e a densidade espacial apresentados estejam subestimados, pela falta de uniformidade na distribuição dos casos, em razão de que apenas uma parcela da população poderia ter buscado atendimento no LEPAC/UEM no período do estudo, representando 17,4\% dos casos do Paraná e $49,6 \%$ dos casos das mesorregiões norte central, centro ocidental e noroeste 25 . Todavia, deve-se considerar que os resultados deste trabalho são mais precisos, em virtude das falhas existentes no processo de notificação por parte dos órgãos responsáveis e, especialmente, por causa do diagnóstico mais preciso da doença e maior capacidade de obtenção de dados epidemiológicos em áreas que servem de base para pesquisas nas mesorregiões em pauta 2,7 .

A transmissão urbana, mais freqüente em Maringá e Cianorte, confirma os resultados de outros trabalhos 2,3,5. Nestes casos, as infecções ocorreram em áreas com preservação de pequenos trechos de cobertura florestal no interior do 
espaço urbano 5 ou no cinturão verde 2,3 . Neste último, a área é dividida em chácaras que servem para a produção de gêneros alimentícios de consumo local, como aves, ovos, frutas, hortaliças e legumes, caracterizando a organização do espaço urbano norte-paranaense 26 . Logo, a leishmaniose tegumentar americana pode se manifestar inclusive na forma de surto, como já ocorreu em Maringá 27. Em áreas de colonização antiga no Paraná, o caráter endêmico da doença tem relação íntima com florestas residuais modificadas ${ }^{2}$. Porém, a adaptação ou pré-adaptação de flebotomíneos e reservatórios silvestres no peridomicílio de zonas rurais e na periferia de zonas urbanas pode estar favorecendo a persistência do ciclo de parasitos do gênero Leishmania, em ambientes com ações antrópicas mais intensas.

Há uma diminuição da densidade dos casos em direção oeste e noroeste, nos interflúvios e mesmo nos vales dos rios Paranapanema, Ivaí e Piquiri, nas suas porções situadas na mesorregião noroeste paranaense. A doença reaparece no extremo noroeste, nos municípios banhados pelo rio Paraná, especialmente em Querência do Norte (7), Porto Rico (7) e Vila Alta (4). Este fato foi verificado anteriormente por Lima et al. 2 . Como existem menos áreas de vegetação nativa preservada na mesorregião noroeste do Paraná, em relação à mesorregião norte central, isto pode interferir na manutenção do ciclo enzoonótico de Leishmania, pela baixa freqüência de reservatórios naturais do parasito.

A distribuição dos casos de leishmaniose tegumentar americana ano a ano mostra uma previsibilidade no comportamento da produ- ção da endemia, com respeito às áreas de ocorrência dos casos. Verificaram-se, no entanto, vários casos isolados e o registro de novas áreas de adensamento, sugerindo novas áreas de produção da endemia. Verificou-se que grande parte dos municípios $(47,4 \%)$ apresentaram de 1 a 3 casos, resultado semelhante ao observado no Estado de São Paulo (54,2\%), conforme Gomes \& Neves 28. Os mesmos autores ressaltam que esse quadro é diferente de situações anteriores, quando predominava a maior concentração de casos em poucos municípios. Fato corroborado neste trabalho, pois nos primeiros anos de notificação da doença, os casos autóctones se restringiam aos municípios do pólo Ivaí, em especial Jussara e Terra Boa.

\section{Conclusão}

A distribuição espacial dos casos de leishmaniose tegumentar americana autóctones do Paraná, conforme a localidade mais provável de infecção, permite definir mais precisamente os municípios da área de abrangência dos pólos de produção da doença, possibilitando a geração de indicadores epidemiológicos e geográficos mais confiáveis do que apenas o número de notificações por município. A distribuição da leishmaniose tegumentar americana no Estado do Paraná sugere a existência de dois circuitos de produção desta doença: circuito Paraná-Paranapanema, onde se destacam os pólos Cinzas-Laranjinha, Tibagi, Ivaí-Pirapó, Piquiri e Baixo Iguaçu, e circuito Ribeira, onde se destaca o pólo Alto Ribeira. 


\section{Resumo}

No Estado do Paraná, a leishmaniose tegumentar americana é endêmica, com 99,3\% dos casos registrados no Sul do Brasil. Verifica-se a distribuição geográfica da doença no norte desse estado, identificando-se as áreas territoriais de maior importância epidemiológica. O estudo foi realizado com dados registrados em fichas epidemiológicas do Laboratório de Ensino e Pesquisa em Análises Clínicas da Universidade Estadual de Maringá, de 1987 a 2004. Consideraram-se apenas os indivíduos que se infectaram nos municípios no norte do Paraná. A identificação das unidades epidemiológicas (pólos e circuitos) foi feita com base na densidade espacial dos casos, conforme o modelo da Fundação Nacional de Saúde, considerando-se as localidades mais prováveis de infecção. De 1.933 casos de leishmaniose tegumentar americana registrados, 1.611 se infectaram em áreas no norte do Paraná. A distribuição da endemia no Estado do Paraná sugere a existência de dois circuitos de produção da doença: circuito Paraná-Paranapanema, onde se destacam os pólos Cinzas-Laranjinha, Tibagi, Ivaí-Pirapó, Piquiri e Baixo Iguaçu, e circuito Ribeira, onde se destaca o pólo Alto Ribeira.

Leishmaniose; Doenças Endêmicas; Doenças Transmissíveis

\section{Referências}

1. Ministério da Saúde. Leishmaniose tegumentar americana: distribuição de casos confirmados de LTA de 1980 a 2005. http://dtr2001.saude.gov.br/ svsepi/situacao_doencas/planilhas_doencas.htm (acessado em 12/Dez/2006).

2. Lima AP, Minelli L, Comunello E, Teodoro U. Distribuição da leishmaniose tegumentar por imagens de sensoriamento remoto orbital, no Estado do Paraná, Sul do Brasil. An Bras Dermatol 2002; 77:681-92.

3. Monteiro WM, Neitzke HC, Lonardoni MVC, Silveira TGV, Ferreira MEMC, Teodoro U. Distribuição geográfica e características epidemiológicas da leishmaniose tegumentar americana em áreas de colonização antiga do Estado do Paraná, Sul do Brasil. Cad Saúde Pública 2008; 24:1291-303.

4. Dias ES, França-Silva JC, Silva JC, Monteiro EM, Paula KM, Gonçalves CM, et al. Flebotomíneos (Diptera: Psychodidae) de um foco de leishmaniose tegumentar no Estado de Minas Gerais. Rev Soc Bras Med Trop 2007; 40:49-52.

5. Teodoro U, Kühl JB, Santos DR, Rodríguez M, Santos ES, Maróstica LM. Flebotomíneos coletados em florestas remanescentes e abrigos de animais silvestres de zoológico no perímetro urbano de Maringá, Sul do Brasil. Estudo preliminar. Rev Soc Bras Med Trop 1998; 31:517-22.

\section{Colaboradores}

U. Teodoro e M. E. M. C. Ferreira orientaram a realização do trabalho e participaram na elaboração do manuscrito. M. V. C. Lonardoni e T. G. V. Silveira contribuíram na elaboração do manuscrito. W. M. Monteiro e H. C. Neitzke contribuíram com a coleta dos dados e na elaboração do manuscrito.
6. Silveira TGV, Arraes SMAA, Bertolini DA, Teodoro U, Lonardoni MVC, Roberto ACBS, et al. Observações sobre o diagnóstico laboratorial e a epidemiologia da leishmaniose tegumentar no Estado do Paraná, Sul do Brasil. Rev Soc Bras Med Trop 1999; 32:413-23.

7. Teodoro U, Silveira TGV, Santos AR, Santos DR, Santos ES, Oliveira O, et al. Freqüência da fauna de flebotomíneos no domicílio e em abrigos de animais domésticos no peridomicílio, nos municípios de Cianorte e Doutor Camargo, Estado do Paraná, Brasil. Rev Patol Trop 2001; 30:209-24.

8. Teodoro U. Características ecológicas de flebotomíneos (Diptera: Psychodidae) em habitats antrópicos, município de Jussara, Paraná, Brasil [Tese de Doutorado]. Curitiba: Universidade Federal do Paraná; 1995.

9. Teodoro U, Santos DR, Santos AR, Oliveira O, Santos ES, Neitzke HC, et al. Avaliação de medidas de controle de flebotomíneos no Município de Lobato, Estado do Paraná, Sul do Brasil. Cad Saúde Pública 2006; 22:415-5.

10. Teodoro U, Santos DR, Santos AR, Oliveira O, Poiani LP, Silva AM, et al. Informações preliminares sobre flebotomíneos do norte do Paraná. Rev Saúde Pública 2006; 40:327-30. 
11. Teodoro U, Balduíno J, Thomaz-Soccol V, Barbosa OC, Ferreira MEMC, Lozovel AL, et al. Environmental sanitation and peridomiciliar organisation as auxiliary practices for the control of phlebotomines in Paraná State, Southern Brazil. Braz Arch Biol Technol 1999; 42:307-14.

12. Teodoro U, Silveira TGV, Santos DR, Santos ES, Santos AR, Oliveira O, et al. Influência da reorganização, da limpeza do peridomicílio e a da desinsetização de edificações na densidade populacional de flebotomíneos no Município de Doutor Camargo, Estado do Paraná, Brasil. Cad Saúde Pública 2003; 19:1801-13.

13. Fundação Nacional de Saúde. Vigilância e monitoramento da leishmaniose tegumentar americana em unidades territoriais-Brasil, 1994-2001. http:// funasa.gov.br (acessado em 05/Fev/2005).

14. Roberto ACBS, Lima AP, Peixoto PR, Misuta NM, Ferreira MEMC, Nerilo-Sobrinho A, et al. Avaliação da terapia com n-metilglucamina e da notificação de leishmaniose tegumentar no noroeste do Estado do Paraná, Brasil. An Bras Dermatol 1997; 72:129-36.

15. Machado LO. A fronteira agrícola no Brasil. In: Becker B, Christofoletti A, Dadidivich FR, Geiger PP, organizadores. Geografia e meio ambiente no Brasil. São Paulo: Editora Hucitec; 1995. p. 35-53.

16. Maack R. Geografia física do Estado do Paraná. 2a Ed. Rio de Janeiro: José Olímpio Editora; 1981.

17. Instituto Paranaense de Desenvolvimento Econômico e Social. Indicadores e mapas temáticos para o planejamento urbano e regional. $\mathrm{Cu}$ ritiba: Instituto Paranaense de Desenvolvimento Econômico e Social; 2003.

18. Pessoa SB, Barreto MP. Leishmaniose tegumentar americana. Rio de Janeiro: Serviço de Documentação do Ministério da Educação e Saúde; 1948.

19. Luz E, Membrive N, Castro EA, Dereure J, Pratlong F, Dedet JA, et al. Lutzomyia whitmani (Diptera: Psychodidae) as vector of Leishmania (V.) braziliensis in Paraná state, southern Brazil. Ann Trop Med Parasitol 2000; 94:623-31.
20. Castro EA, Thomaz-Soccol V, Membrive N, Luz E. Estudo das características epidemiológicas e clínicas de 332 casos de leishmaniose tegumentar notificados na região norte do Estado do Paraná de 1993 a 1998. Rev Soc Bras Med Trop 2002; 35: 445-52.

21. Teodoro U, La Salvia Filho V, Lima EM, Misuta NM, Verzignassi TG, Ferreira MEMC. Leishmaniose tegumentar: flebotomíneos de área de transmissão na região norte do Paraná, Brasil. Rev Saúde Pública 1991; 25:129-33.

22. Velasquez LG, Membrive N, Membrive $U$, Rodrigues G, Reis N, Lonardoni MVC, et al. PCR in the investigation of canine American tegumentary leishmaniasis in northwestern Paraná State, Brazil. Cad Saúde Pública 2006; 22:571-8.

23. Zanzarini PD, Santos DR, Santos AR, Oliveira O, Poiani LP, Lonardoni MVC, et al. Leishmaniose tegumentar americana canina em municípios do norte do Estado do Paraná, Brasil. Cad Saúde Pública 2005; 21:1957-61.

24. Fundação Nacional de Saúde. Manual de controle da leishmaniose tegumentar americana. http:// funasa.gov.br (acessado em 12/Jul/2007).

25. Ministério da Saúde. Sistema Nacional de Vigilância em Saúde: relatório de situação - Paraná. http://portal.saude.gov.br/portal/arquivos/pdf/ pr1.pdf (acessado em 14/Ago/2007).

26. Companhia Melhoramentos do Norte do Paraná Colonização e desenvolvimento do Norte do Paraná. São Paulo: Companhia Melhoramentos do Norte do Paraná; 1975.

27. Silveira TG, Roberto ACBS, Zanzarini PD, Venazzi EAS, Mangabeira HN, Teodoro U, et al. Leishmaniose tegumentar americana: surto na região urbana, no município de Maringá, Norte do Paraná. Rev Soc Bras Med Trop 2004; 37 Supl III:49.

28. Gomes AC, Neves VLFC. Estratégia e perspectivas de controle da leishmaniose tegumentar no Estado de São Paulo. Rev Soc Bras Med Trop 1998; 31:553-8.

Recebido em 14/Abr/2008

Versão final reapresentada em 02/Dez/2008

Aprovado em 21/Jan/2009 